\title{
CBIR - An analysis and suggestions for improvement
}

\author{
Shriram K V \\ Research Scholar, PRIST \\ University, \\ Tanjore, Tamilnadu, India
}

\author{
P.L.K.Priyadarsini \\ Associate Professor, \\ Department of Computer \\ Science, \\ PRIST University, Tanjore \\ Tamilnadu, India,
}

\author{
Subashri V \\ M.Tech (II Year), C.S.E Dept, \\ B.S.Abdur Rahman University, \\ Chennai, Tamilnadu, India
}

\begin{abstract}
The basic motive of the search engines lies in the fact that, it should be able to retrieve results with utmost accuracy and efficiency. Image search engines are no where exceptional. They should retrieve images from the database which are similar to the query image. Existing image search engines largely depend on the keywords provided by the users for effective search. In this paper, we have discussed the disadvantages of keyword based search as well as the basics of Content Based Image Retrieval (CBIR). In particular, we discuss the pros and cons of CBIR based on Color and Texture properties of the images and suggest a new parameter for search, the entropy.
\end{abstract}

\section{General Terms}

Image Processing, CBIR, Image Retrieval.

\section{Keywords}

Image Processing, CBIR, Histogram, Wavelet decomposition, Euclidean distance, Quadratic distance, Entropy.

\section{INTRODUCTION}

Content Based Image retrieval has seen so many changes over the last decade. Most of them were based on the combination of color, texture and shape properties of an image. Some of the recently suggested CBIR systems include relevance feedback from the user for filtering the images. Though several changes have been suggested, only a very few parameters, including, the color and texture have been taken into account and the CBIR systems were developed based on them. As a result, the expected outcome is never achieved by the existing systems. Irrelevant images dominate the relevant images. In order to perform better, most of the existing systems expect keywords from the users to go on for a keyword based search. But, keyword based search proves to be inefficient, since, it forces the database admin to name every image manually according to the subject of the image. If the image is named without considering the subject, keyword based subject will fail at the first step itself. Hence, we have suggested a randomness parameter called the entropy to be used along with the basic parameters, namely, the color and the texture. The following section gives a basic introduction about the properties of images, followed by the technical background of the implementation.

\section{ANALYSIS OF THE EXISTING SYSTEMS}

The basic steps involved in CBIR are explained in the figure 1 . The objective is to enter an image as a query image and the resulting images should be similar to that of the query image.
The basic property used in comparing images is the color combination of the query image. The following sections deal with the intrinsic details of color based image retrieval and texture based image retrieval.

\subsection{Color based image retrieval}

The basic tool in analyzing the colors present in an image is the histogram. They are bar graph like thing, where, the bars are termed as bins. Number of bins is proportional to the number of colors that make up the image file. For example, if the image is composed of Red, Green and Blue alone, there will be 3 bins in the x-axis. The y-axis, on the other hand describes the number of pixels which are of the particular color. For example, refer to the figure 2 shown below. A sample image with its histogram is shown.

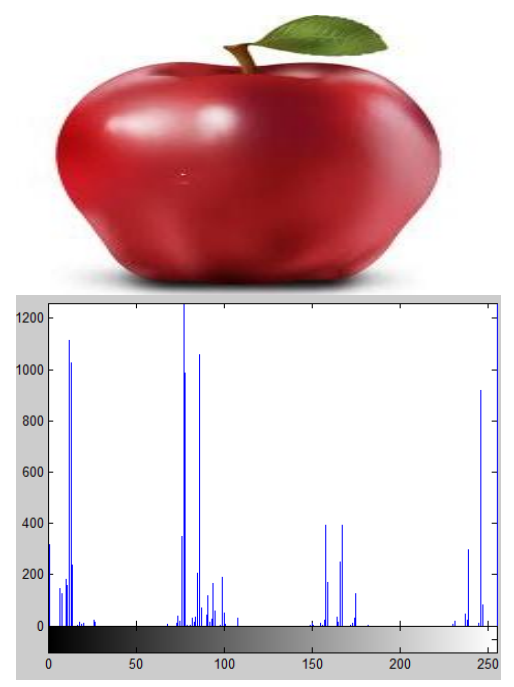

Fig 2: Sample Image and its histogram

Based on the histograms, a similarity check is performed. Generally images are of RGB color maps. They are converted into HSV maps for convenience. The steps for image comparisons are follows.

- Obtain the query image from the user. Generate the histogram of the image.

- Do the above step for every database image. 


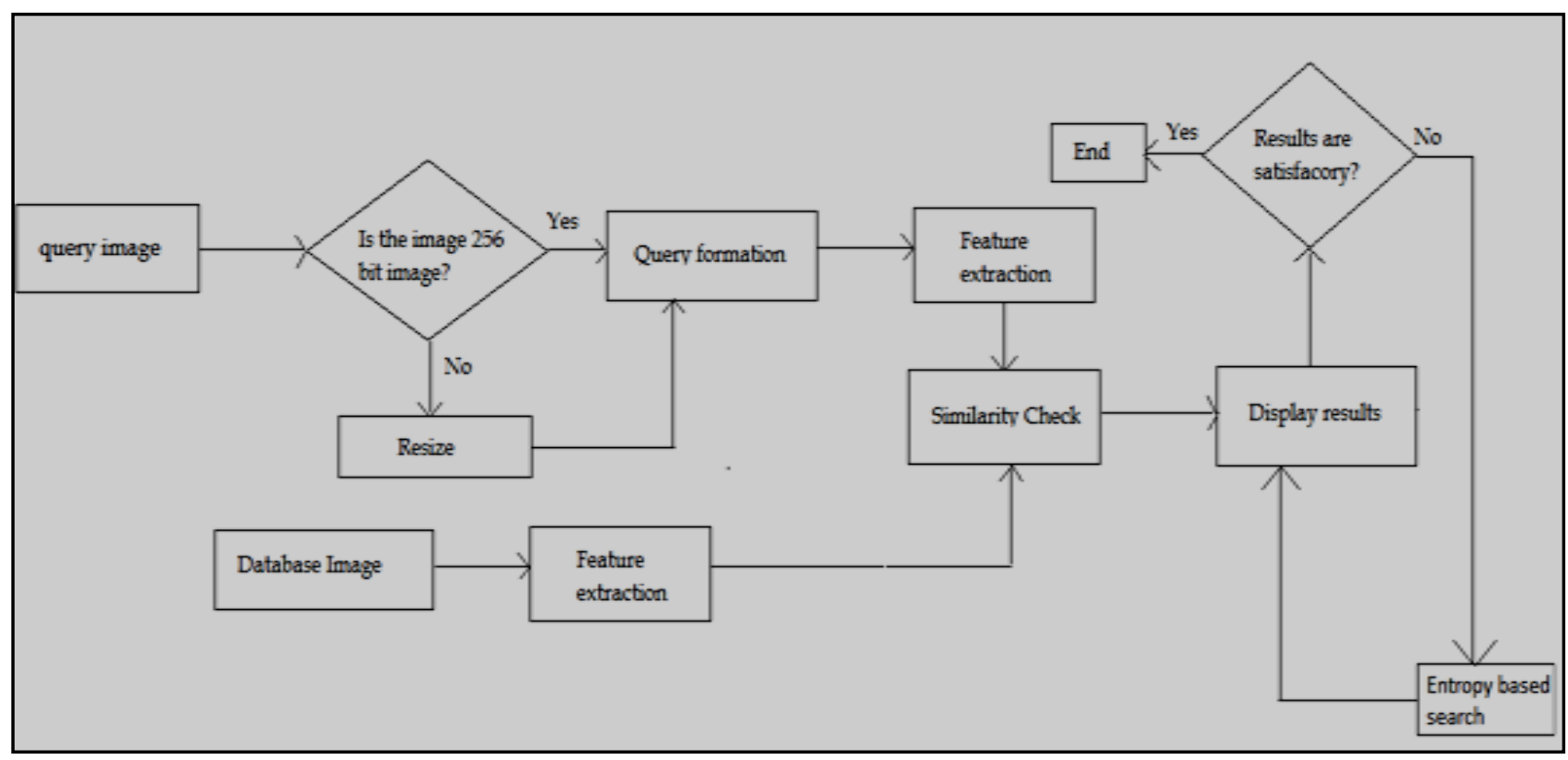

Fig 1: Basic steps in CBIR

- Compute the quadratic distance between the query image and every database image according to the formula $^{[1]}$,

$$
d^{2}=\left(H_{Q}-H_{I}\right)^{t} A\left(H_{Q}-H_{I}\right)
$$

Where, $\mathrm{d}$ is the measured quadratic distance between the query image $(\mathrm{Q})$ and the database image (I), $\mathrm{H}_{\mathrm{Q}}$ and $\mathrm{H}_{\mathrm{I}}$ are the histograms of $\mathrm{Q}$ and $\mathrm{I}$ respectively, ' $\mathrm{t}$ ' is the transpose operation, and ' $\mathrm{A}$ ' denotes the similarity matrix, which is computed based on the Hue, Saturation and Value of the images being handled. The formula to find the similarity matrix is as follows ${ }^{[1]}$,

$\boldsymbol{A}_{(i, j)}=$

$1-\frac{1}{\sqrt{5}}\left[\left(v_{q}-v_{i}\right)+\left(s_{q} \cos \left(h_{q}\right)-s_{i} \cos \left(h_{i}\right)^{2}+\right.\right.$

$\left.s_{q} \sin \left(h_{q}\right)-s_{i} \sin \left(\left(h_{i}\right)\right)^{2}\right]^{\frac{1}{2}}$

- Based on the value of quadratic distance between the images, the results are prioritized. Lesser the value of ${ }^{d}$ ', greater the possibility of similarity.

A sample query and the retrieval results are shown in figure 3 . As it can be observed from the figure shown, the color based image retrieval proves to be somewhat efficient. The search result has some irrelevant images too. In order to bring down the irrelevancy rate, one more level of search, filtering the irrelevant images needs to be done. The following section deals with the texture based image retrieval.

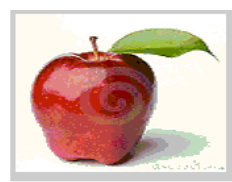

Query Image

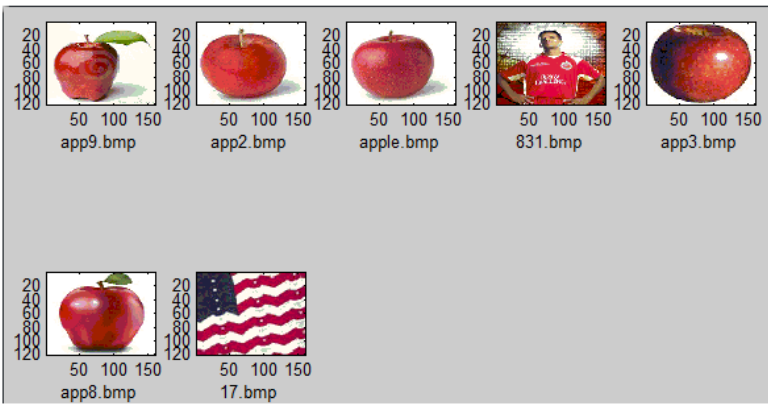

Color search results

Fig 3: Sample query and search results

\subsection{Texture based image retrieval}

To improve the retrieval efficiency of CBIR systems, images were analyzed deeply. The objective of this step is to calculate the overall intensity of the images, which can then be used as a measure for comparison. The coarser information about the images is obtained by decomposing the images, using wavelet decomposition method. The following steps are followed for the texture based search.

- Top 6 energies of the image (Query image and every database image) according to the formula ${ }^{[2]}$,

$$
E=\frac{1}{M N} \sum_{i=1}^{M} \sum_{j=1}^{N}|X(i, j)|
$$

Where, ' $\mathrm{M}$ ' and ' $\mathrm{N}$ ' are the row and column size of the decomposed image. For instance, consider the image in the figure 4 . 


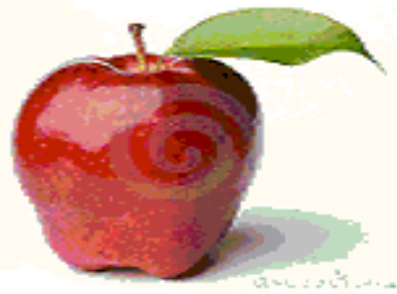

Fig 4: Image to be decomposed

The first step is to decompose the image, using discrete wavelet transformation function dwt2() available in MatLab, the parameters of the function are matrix of pixels of image that is to be decomposed and the type of the wavelet. We preferred Daubechies wavelet; in particular, we tried db10 for testing purposes. Figure 5 shows first level decomposition of the image shown in figure 4.

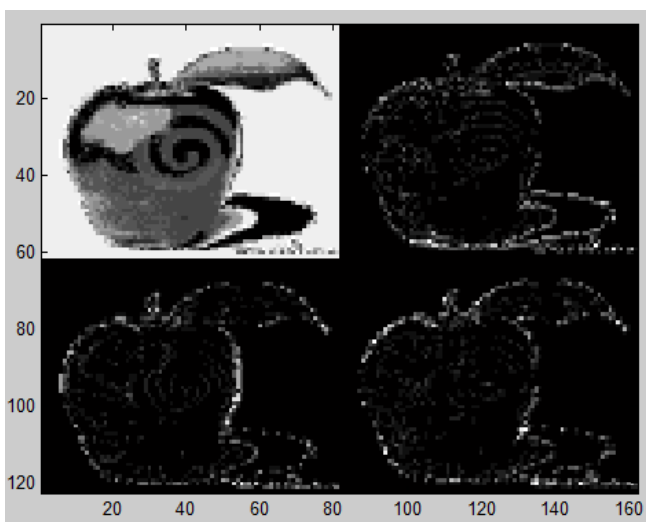

Fig 5: First Level decomposition

As the above figure shows, the image is decomposed at different frequency bands, where in the energy is concentrated in the low-low sub band

It is because of this property, we utilized the lowlow sub band for the further 5 levels of decompositions. At the end of $6^{\text {th }}$ level of decomposition, we calculated the energy using (3).

- The above step is repeated for every image in the database, and finally, we calculated the Euclidean distance between the images using the following formula ${ }^{[2]}$,

$$
D_{i}=\sum_{k=1}^{k}\left(X_{k}-Y_{i, k}\right)^{2}
$$

Where Di denotes the Euclidean distance between the query image and ith image in the database. [Note: For every image $\mathrm{i}$ in the database, $\mathrm{k}$ set of energies have to be calculated. $\left.\left(\mathrm{Y}_{\mathrm{i}, \mathrm{k}}\right)\right] \mathrm{X}_{\mathrm{k}}$ is the energy level of the query image. Finally we sorted the distance values in the increasing order and displayed the top most images.

- Based on the value of D, we sorted the images in the database in the descending order to provide the most similar images at the top. A sample query and the retrieval results are shown in figure 6 .

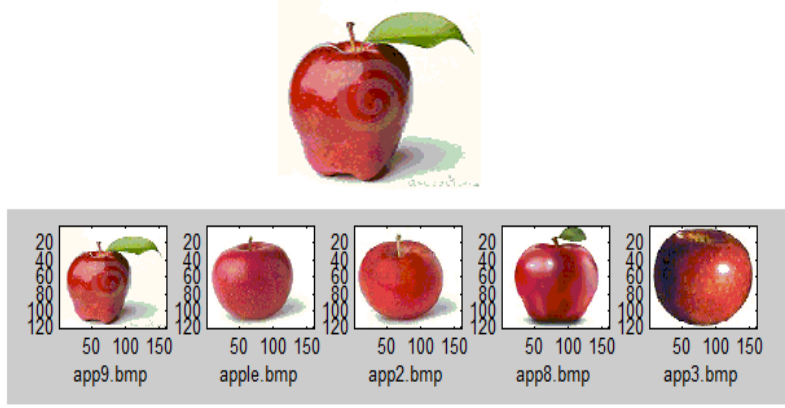

Fig 6: Sample query and search results

It is evident from the figure 6 that, the search results have been much convincing than that of the color based retrieval. Irrelevant images are filtered to a great extent in texture based retrieval.

\section{RECOMMENDATION}

Our test results suggested us that, it is not sufficient to use only the color and texture properties of an image to implement CBIR, since, the efficiency was not as expected. Hence, we made a profound study of image properties that can help us figure out the objective. We have identified a very useful property, namely, the entropy, which seemed to be highly constraining the search results, by completely eliminating the irrelevant images.

\subsection{Entropy}

Entropy is a factor that is used to characterize the randomness of an image. Entropy is a statistical measure of randomness that can be used to characterize the texture of the input image $^{[3]}$. Entropy can be defined as follows'

$$
-\operatorname{sum}\left(p \cdot \log _{2}(p)\right.
$$

The ' $\mathrm{p}$ ' in the above term defines the histogram count of the image being processed. Figure 7 shows an image and its entropy value.

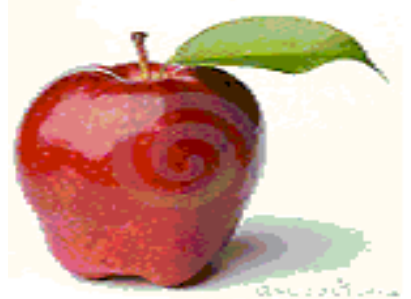

\subsection{2}

\section{Fig 6: Images and their entropy}

We are working towards utilizing the entropy value of the query image and the images in the database, in order to compare them. We are on the analysis phase of choosing the right threshold of the entropy difference between the images to minimize the percentage of irrelevance.

\subsection{Region Of Interest (ROI)}

We are planning to further improve the search criterion based on some region of interest chosen by the user. The user will have to choose a region in the query image to insist upon the particular pattern as a symbol of training the system. The 
system will then have to work on the particular region. The region will be analyzed based on the histogram properties and the entropy properties, to revise the query. After the entropy based search is over, this part of the research will be taken up.

\section{ACKNOWLEDGMENTS}

We wish to thank the university and the faculties for providing the consistent support for getting this research work going on.

\section{REFERENCES}

[1] Kondekar V. H., Kolkure V. S., Kore S.N. "Image Retrieval Techniques based on Image Features: A State of Art approach for CBIR", International Journal of Computer Science and Information Security,Vol. 7, No. 1,2010 .

[2] Rahul Metha, Nishkhol Mishra, Sanjeev Sharm, "Color Texture based image retrieval system", International Journal of Computer Applications (0975 - 8887), Volume 24 - No. 5 June 2011.

[3] MatLab Manual.

[4] http://pages.cs.wisc.edu/ beechung/docs/papers.html

[5] http://www.ee.columbia.edu/ jrsmith/html/pubs/tatfcir/n ode22.html

[6] A.M.W. Smeulders, M. Worring, S. Santini, A.Gupta, and R. Jain, "Content-based image retrieval at the end of early years, "IEEE Trans. On Pattern Analysis and machine intelligence, vol. 22, no. 12, december 2000.

[7] Satya Sai Prakash, RMD. Sundaram. "Combining Novel features for Content Based Image Retrieval". IEEE XPLORE DIGITAL LIBRARY pages: 373 - 376, 12 November 2007.

[8] Ja-Hwung Su, Wei-Jyun Huang, Philip S. Yu, and Vincent S. Tseng, "Efficient Relevance Feedback for Content-Based Image Retrieval by Mining User Navigation Patterns" IEEE transactions on knowledge and data engineering, vol. 23, no. 3, march 2011.

[9] Liana Stanescu, IADIS International Conference on Applied Computing, 2005 "on-line software system for content-based visual query of a color medical imagery."

[10] Neetesh Gupta, R.K.Singh, "A New Approach for CBIR Feedback based Image Classifier", International Journal of Computer Applications (0975 - 8887) Volume 14No.4, January 2011.

[11] Wasim Khan, Shiv Kumar. Neetesh Gupta, Nilofar Khan, "A Proposed Method for Image Retrieval using Histogram values and Texture Descriptor Analysis", International Journal of Soft Computing and Engineering (IJSCE) ISSN: 2231-2307, Volume-I Issue-II, May 2011.

[12] Gonzalez, R.C., Woods, R.E, "Digital Image Processing” 2nd Ed., Prentice Hall. 